\title{
Biosynthesis of Salvianic Acid from L-dopa via a Two-Step Process
}

\author{
Weirui Zhao ${ }^{1}$, Keshun Hu${ }^{1}$, Jiaqi Mei ${ }^{2}$, Lehe Mei ${ }^{1 *}$ \\ ${ }^{1}$ School of Biotechnology and Chemical Engineering, Ningbo Institute of Technology, Zhejiang University, \\ 1\#, Qianhu South Road, Ningbo, China \\ zhaowrzju@zju.edu.cn; 1184844153@qq.com; meilh@zju.edu.cn \\ ${ }^{2}$ Hangzhou Zhongmei Huadong Pharm. Co. Ltd \\ 866\#, Moganshanlu, Hangzhou, China \\ 346671416@qq.com
}

\section{Extended Abstract}

Salvianic acid A (SAA, 3-(3',4'-dihydroxyphenyl)-2-hydroxypropanoic acid), as the main bioactive component of traditional Chinese herb Salvia miltiorrhiza, has important application value in the treatment of cardiovascular diseases ${ }^{[1]}$. Traditionally, SAA was mainly isolated from dried root of $S$. miltiorrhiza by a water-extraction process. However, the amount of SAA in crude root is very low $(0.045 \%)$, which restricted its large-scale applications. Although several chemical methods for SAA synthesis have been developed, these methods also suffered from intractable enantioselectivities, complicated procedure, and environmental pollution ${ }^{[2]}$. Therefore, developing efficient and eco-friendly methods for SAA production was highly desirable. In this study, a two-step biocatalytic cascade reaction to produce SAA from inexpensive L-dopa with high efficiency by using whole-cell biocatalysts was developed. In the first step, the recombinant Escherichia coli cells expressing mL-AAD from Proteus vulgaris (BL21(DE3)-pET-28a-mlaad) were employed to deaminize L-dopa to form 3,4dihydroxyphenylalanine (DHPPA). Subsequently, the permeabilized recombinant $E$. coli cells co-expression of d-lactate dehydrogenase (d-LDH) from Pediococcus acidilactici and FDH from Mycobacterium vaccae N10 (NADH regeneration system) (BL21(DE3)-pETDuet- $p d d h-f d h)$ were used to convert DHPPA in the raw reaction solution to SAA.

Results: (1) The effect of bioconversion conditions for BL21(DE3)-pET-28a-mlaad to convert L-dopa to DHPPA were evaluated firstly. The optimal conditions for the deamination reaction were as follows: $0.42 \mathrm{~g} / \mathrm{L}$ cell biomass, $50 \mathrm{mM} \mathrm{L-}$ dopa, $37^{\circ} \mathrm{C}$, pH 7.5 and 160 min (operation time). (2) To overcome the permeability barrier of cell envelope to substrates and products, the hexane-permeabilized BL21(DE3)-pETDuet-pddh-fdh was used to convert DHPPA to SAA. The optimal $\mathrm{pH}$ and temperature for the reaction were 6.0 and $30^{\circ} \mathrm{C}$, respectively. (3) Two-step catalytic synthesis of SAA from L-dopa: DHPPA was prepared with BL21(DE3)-pET-28a-mlaad under the optimum deamination conditions described above, and 48.6 mM DHPPA was obtained from $50 \mathrm{mM}$ L-dopa. Next, the recombinant BL21(DE3)-pET-28a-mlaad cells were removed from the reaction solution by centrifugation, $100 \mathrm{mM}$ sodium formate were added and the reaction $\mathrm{pH}$ value was adjusted to 5.5. Then $0.31-0.93 \mathrm{mg} / \mathrm{mL}$ permeabilized BL21(DE3)-pETDuet- $p d d h-f d h$ were added to the reaction solution to start the conversion of DHPPA to SAA. The yields of SAA from DHPPA in our experimental ranges could all reach more than $97.7 \%$, and the SAA production rate was accelerated with increasing cell concentration. When cell concentration above $0.62 \mathrm{mg} / \mathrm{mL}$, DHPPA could almost be converted to SAA in $4.5 \mathrm{~h}$. In case of $0.31 \mathrm{mg} / \mathrm{mL}$ cell concentration, SAA production rate from DHPPA could over $97.7 \%$ after $5.5 \mathrm{~h}$. Overall, in our developed a two-step biotransformation process, L-dopa was efficiently deaminized to DHPPA with a high yield of $97.7 \%$ in mL-AAD bioconversion, then DHPPA was effectively converted to SAA by the permeabilized recombinant $E$. coli cells co-expression of d-LDH and FDH. The total yield of SAA from L-dopa could reach approximately $96.5 \%$ by the two-step biocatalytic reaction in the optimum reaction conditions.

Therefore, the methods developed herein provided excellent production efficiency and reflected good industrial application prospects.

\section{References}

[1] G. Zhao, H. Zhang, T. Ye, et al, "Characterization of the radical scavenging and antioxidant activities of danshensu and salvianolic acid B," Food Chem. Toxicol., vol. 46, no. 1, pp. 73-81, 2008.

[2] Y. Bai, Q. Zhang, P. Jia, et al, "Improved process for pilot-scale synthesis of danshensu ((+/-)-dss) and its enantiomer derivatives," Org. Process Res. Dev., vol. 18, pp. 1667-1673, 2014. 